\title{
Silicose Classique Et Compliquée Chez Deux Jeunes Frères Lapidaires
}

\section{Oninala Fenitra Rakotondrasoa,}

Service de Pneumologie - CHU Joseph Ravoahangy Befelatanana,

Faculté de Médecine- Université d'Antananarivo

Kiady Ravahatra,

Service de Pneumologie - CHU Fenoarivo,

Faculté de Médecine-Université d'Antananarivo

Michel Harison Tiaray, Arnauld Martin Fidy, Anjara Mihaja Nandimbiniaina,

Service de Pneumologie - CHU Joseph Ravoahangy Befelatanana,

Faculté de Médecine- Université d'Antananarivo

\section{Marie Odette Rasoafaranirina,}

Service de Pneumologie CHU Tambohobe Fianarantsoa,

Faculté de Médecine- Université d'Antananarivo

Iantsotiana Davidson Rakotondrabe,

Service de Pneumologie - CHU Joseph Ravoahangy Befelatanana,

Faculté de Médecine- Université d'Antananarivo

\section{Jobeline Rajaoarifetra,}

Service des Maladies cardio-respiratoires - Hopital Manaram-Penitra

Tamatave, Faculté de Médecine - Université d'Antananarivo

\section{Jocelyn Robert Rakotomizao, Joelson Lovaniaina Rakotoson,}

Service de Pneumologie - CHU Joseph Ravoahangy Befelatanana,

Faculté de Médecine- Université d'Antananarivo

Rondro Nirina Raharimanana,

Service de Pneumologie - CHU Fenoarivo,

Faculté de Médecine-Université d'Antananarivo

\section{Résumé}

Les maladies professionnelles, qui se manifestent surtout chez les mineurs et les lapidaires, ne sont pas encore maîtrisées à Madagascar. Les conditions de travail et la pauvreté constituent des facteurs de gravité des pneumoconioses. Dans cet article deux cas de jeunes frères lapidaires sont 
rapportés, atteints d'une silicose diagnostiquée au stade tardif reflétant cette réalité. La symptomatologie était dominée par une dyspnée chronique d'aggravation progressive compliquée d'une insuffisance respiratoire chronique et d'un cœur pulmonaire chronique chez les deux patients. Les investigations étaient limitées par le manque de moyens financiers. La radiologie a montré une image de miliaire pour le premier patient, et une atélectasie de deux lobes pulmonaires à droite pour le deuxième. Le traitement était basé sur l'usage de corticoïdes, de bronchodilatateurs et surtout sur l'oxygénothérapie de longue durée. Leur évolution clinique a été favorable au début de l'hospitalisation mais s'est détériorée au bout de quelques semaines. La silicose est une affection grave, notamment par ses complications. À Madagascar, le système de santé jouerait un grand rôle dans sa prévention.

Mots-clés : Silicose, Classique, Jeunes, Madagascar 


\title{
Complicated and Classical Silicosis in Two Young Stonecutters Brothers
}

\section{Oninala Fenitra Rakotondrasoa,}

Service de Pneumologie - CHU Joseph Ravoahangy Befelatanana,

Faculté de Médecine- Université d'Antananarivo

Kiady Ravahatra,

Service de Pneumologie - CHU Fenoarivo,

Faculté de Médecine-Université d'Antananarivo

Michel Harison Tiaray, Arnauld Martin Fidy, Anjara Mihaja Nandimbiniaina,

Service de Pneumologie - CHU Joseph Ravoahangy Befelatanana,

Faculté de Médecine- Université d'Antananarivo

\section{Marie Odette Rasoafaranirina,}

Service de Pneumologie CHU Tambohobe Fianarantsoa,

Faculté de Médecine- Université d'Antananarivo

Iantsotiana Davidson Rakotondrabe,

Service de Pneumologie - CHU Joseph Ravoahangy Befelatanana,

Faculté de Médecine- Université d'Antananarivo

\section{Jobeline Rajaoarifetra,}

Service des Maladies cardio-respiratoires - Hopital Manaram-Penitra

Tamatave, Faculté de Médecine - Université d'Antananarivo

\section{Jocelyn Robert Rakotomizao, Joelson Lovaniaina Rakotoson,}

Service de Pneumologie - CHU Joseph Ravoahangy Befelatanana,

Faculté de Médecine- Université d'Antananarivo

Rondro Nirina Raharimanana,

Service de Pneumologie - CHU Fenoarivo,

Faculté de Médecine-Université d'Antananarivo

\begin{abstract}
Occupational diseases, which occur especially among mineworkers and stonecutters, are yet to be controlled in Madagascar. Working conditions and poverty are factors of pneumoconiosis gravity. This paper focuses on two cases of two young stonecutters' brothers affected by a silicosis diagnosed at
\end{abstract}


a late stage, reflecting this reality. The symptomatology was dominated by a chronic dyspnea with gradual worsening, complicated by chronic respiratory failure and chronic cor pulmonale in the two patients. The investigations were limited by the lack of financial resources. Radiology showed miliary for the first patient and two lobes' atelectasis for the second patient. The treatment was based on the use of corticoids and bronchodilators, most especially, on long term oxygen therapy. Their clinical evolution was successful at the beginning of their hospitalization but later worsened after some weeks. Silicosis is a serious condition, particularly by its complications. In Madagascar, the health care system aims to play a significant role in its prevention.

Keywords: Silicosis, Classical, Young, Madagascar

\section{Introduction}

La silicose est une affection appartenant à la classe des maladies pulmonaires professionnelles résultant de l'inhalation de dioxyde de silicium et d'une réaction correspondante du tissu pulmonaire, pouvant induire des lésions irréversibles sur ce tissu (International Labour Organization (ILO), 1971). Il s'agit d'une pathologie fréquemment rencontrée chez les personnes exerçant des activités dans les domaines suivants : forage, tunnellisation, carrière, traitement des pierres ou de matériaux contentant du quartz (Ziskind, Jones \& Weill, 1976). À l'échelle mondiale, la silicose constitue encore une maladie dont les facteurs de risque et les effets sur la santé restent encore difficiles à éliminer complètement. Selon l'OMS en 2002, l'exposition à des polluants particulaires présents dans l'air des lieux de travail était responsable chaque année de 386000 décès, dont 30.000 dans le cadre des pneumoconioses (Fedotov \& Eijkemans, 2007). Bien que la prévalence de la silicose à Madagascar ne soit pas encore bien définie, elle y demeure un problème de santé publique.

Malgré le fait que les activités professionnelles soient régies par les lois du travail, la plupart des mineurs et des lapidaires Malgaches n'utilisent pas de dispositifs de protection en travaillant, ou dans d'autres situations, font usage d'équipements ou de locaux inadéquats, ne permettant pas de réduire l'émission des particules nuisibles à la santé. Ces travailleurs sont ainsi exposés à des maladies professionnelles, dont la silicose. À Madagascar, ces pathologies sont souvent diagnostiquées au stade tardif, rendant ainsi la prise en charge difficile. Les objectifs de cette étude sont alors de rapporter deux cas de silicoses diagnostiquées tardivement chez deux jeunes frères lapidaires vus au Centre Hospitalier Universitaire de Fenoarivo afin d'illustrer cette situation existant à Madagascar, et de souligner l'importance du suivi respiratoire chez les Malgaches exposés à la silice. 


\section{Observation}

Le premier cas (Sujet A), âgé de 30 ans, a été hospitalisé pour une dyspnée d'effort d'aggravation progressive, devenue de plus en plus invalidante. Dans ses antécédents, une tuberculose pulmonaire, traitée et déclarée guérie, a été relevée, compliquée au cours du traitement même par un pneumothorax spontané, qui a été drainé avec une bonne évolution. C'est un patient lapidaire travaillant les cristaux de quartz rose depuis 10 ans, sans utilisation de masque de protection. À l'examen, il a présenté un syndrome de détresse respiratoire aigüe avec une désaturation périphérique en oxygène $\left(\mathrm{SpO}_{2}=73 \%\right.$ à l'air ambiant). La radiographie du thorax a objectivé une atélectasie du poumon droit avec des opacités alvéolo-interstitielles gauches et une cardiomégalie au dépens du cœur droit (Figure 1).

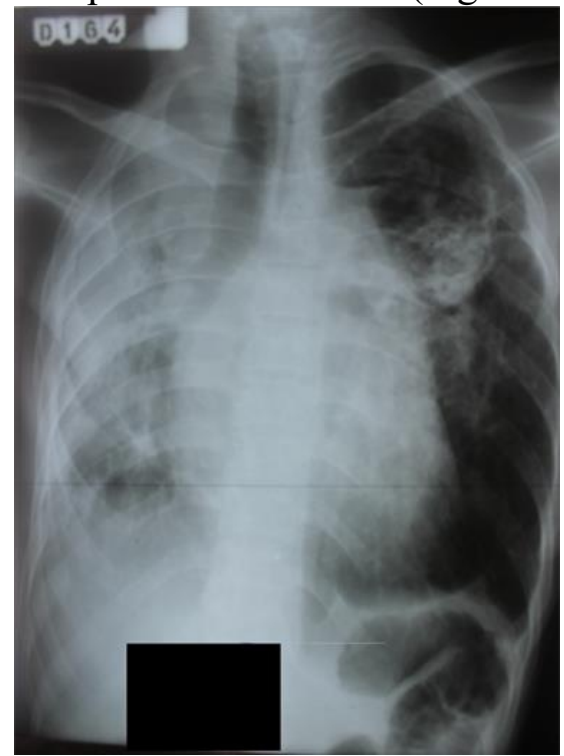

Figure 1. Radiographie du thorax de face du Sujet A à l'admission. Classification des lésions radiologiques selon la classification du BIT : Champ pulmonaire droit : 2/3, LU, q/t, cg (nodule non pneumoconiotique), ra (atélectasie). Champ pulmonaire gauche : opacité appartenant à la catégorie C. Médiastin : co (cardiomégalie).

L'électrocardiogramme a montré une tachycardie régulière avec une fréquence cardiaque à 115 battements/minute et une hypertrophie des cavités droites. Le scanner thoracique et l'échographie Doppler cardiaque n'ont pas été réalisés par faute de moyens financiers. La spirométrie a montré un trouble ventilatoire mixte à prédominance obstructive. La recherche de Bacilles Acido-Alcoolo-Résistants (BAAR) dans les crachats était revenue négative. Le patient a été traité par une oxygénothérapie, une corticothérapie à 1 $\mathrm{mg} / \mathrm{kg} /$ jour et un traitement par bronchodilatateurs et par des antibiotiques. L'évolution a été favorable au début avec une amélioration de la saturation 
périphérique en oxygène, suivie d'une détérioration progressive de son état respiratoire.

Le deuxième cas (Sujet B) était un homme âgé de 27 ans qui a présenté une dyspnée d'aggravation progressive associée à une toux productive évoluant depuis 4 mois. Elle a été traitée en premier lieu par un médecin de ville comme une miliaire tuberculeuse devant une image de miliaire radiologique selon le schéma thérapeutique de l'OMS comportant 2 mois de Rifampicine, Isoniazide, Éthambutol et Pyrazinamide et 4 mois de Rifampicine et Isoniazide. Malgré ce traitement, une aggravation de son état clinique et de l'image radiologique a été notée ; c'est-à-dire, des micronodules qui deviennent confluents, une augmentation de leur taille, ainsi que l'apparition d'un pneumothorax spontané. Il s'agit également d'un patient lapidaire travaillant les cristaux de quartz rose depuis 10 ans, sans utilisation de masque de protection comme son frère. L'examen clinique à l'entrée a révélé un syndrome de détresse respiratoire aigüe, une désaturation périphérique ( $\mathrm{SpO} 2$ à $82 \%$ à l'air ambiant), un syndrome d'épanchement pleural gazeux apical gauche et des signes d'insuffisance cardiaque droite. À la radiographie du thorax, des opacités micronodulaires diffus à répartition symétrique ont été objectivées au niveau des deux champs pulmonaires, un pneumothorax au niveau de la région apicale droite et une hyperclarté apicale gauche (Figures 2-4).

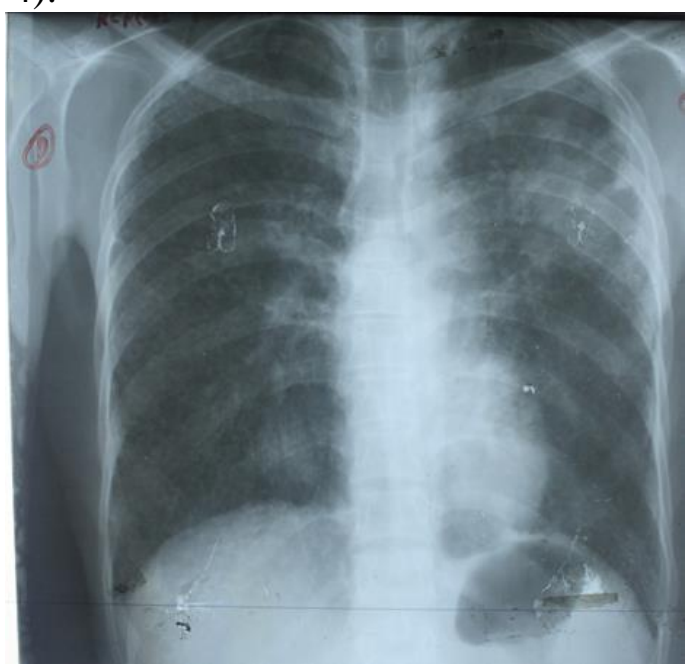

Figure 2. Radiographie du thorax de face du Sujet B à 8 mois avant l'admission.

Classification des lésions radiologiques selon la classification du BIT : Lésions de type 3/+ diffus, $p$. 


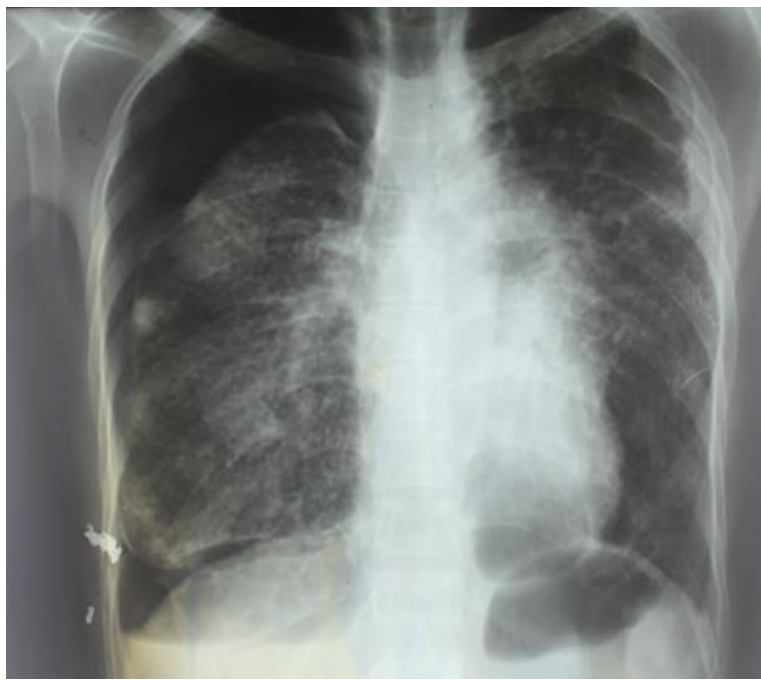

Figure 3. Radiographie du thorax de face du Sujet B à 5 mois avant l'admission. Classification des lésions radiologiques selon la classification du BIT : Lésions de type 2/2 diffus, q. Champ pulmonaire droit : px (pneumothorax).

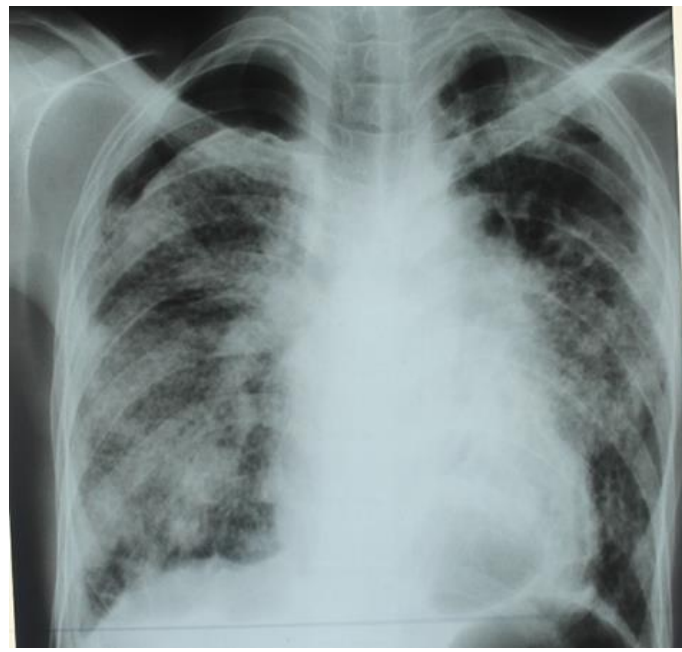

Figure 4. Radiographie du thorax du Sujet B à l'admission. Classification des lésions radiologiques selon la classification du BIT : Lésions 3/2 diffus, q/t. Champ pulmonaire droit : opacités larges catégorie A, px (pneumothorax). Médiastin : co (cardiomégalie). Champ pulmonaire gauche : em (emphysème).

L'ECG a montré un signe d'hypertrophie des cavités droites. Le scanner thoracique et l'échographie Doppler cardiaque n'ont pas été réalisés par faute de moyens financiers. La recherche de BAAR dans les crachats ainsi que la technique de biologie moléculaire (GeneXpert MTB/RIF) étaient négatifs. Un traitement par une oxygénothérapie, une corticothérapie et par des bronchodilatateurs a été instauré. L'évolution était stationnaire, marquée 
par des épisodes de détresses respiratoires aigües et une dépendance à l’oxygène.

Au total, pour les deux cas le diagnostic retenu a été celui de silicose classique chez des sujets jeunes, compliquée d'insuffisance respiratoire chronique restrictive probable et de cœur pulmonaire chronique.

\section{Discussion}

Les cas décrits ci-dessus illustrent bien les situations existant à Madagascar concernant ces maladies professionnelles ; c'est-à-dire, la non utilisation de matériels de protection au cours du travail et la consultation chez un spécialiste uniquement au stade tardif de la maladie avec un pronostic fonctionnel mauvais, rendant la prise en charge difficile.

Nos deux patients sont des frères âgés respectivement de 27 et de 30 ans ; c'est-à-dire, plus jeunes que ceux rapportés dans deux études africaines chez des sujets silicotiques : respectivement de 36 ans et de 32,2 ans (Hassinet et al., 2006 ; Senhaji et al., 2015). En Chine, Han et al (2019) ont décrit une moyenne d'âge de diagnostic significativement élevée à la nôtre (61 ans). Cela pourrait être expliqué par le fait que les deux frères travaillaient à un âge très précoce comparé à celui de la majorité décrite dans la littérature. La durée d'exposition de nos patients est de 9 ans. Dans la littérature, des durées d'exposition plus longues sont rapportées pour la silicose (Senhaji et al., 2015 ; Han et al., 2019 ; Elidrissi et al., 2016 ; Elkard et al., 2016). Toutefois, Hassinet et al. (2006), ainsi que Heddane et al. (2019) ont décrit des durées moyennes d'exposition très courtes respectivement : 2 ans, 1 à 4 ans. Plus la quantité de particules inhalées est grande, et que les infrastructures utilisées sont inadéquates au cours du travail, plus le délai d'apparition de la maladie est court. Par ailleurs, comme il s'agit ici de deux frères, une explication du point de vue génétique n'est pas à écarter.

Sur le plan clinique, les patients étudiés ont présenté le même signe qui est souvent décrit lors des silicoses ; c'est-à-dire, la dyspnée chronique (Senhaji et al., 2015 ; Heddane et al., 2019).

Selon la classification du Bureau International du Travail (BIT), les anomalies radiologiques se présentent soit sous forme de petites opacités plus ou moins arrondies de type « $\mathrm{p}$ », lorsque le diamètre est inférieur à $1.5 \mathrm{~mm}$, de type « $\mathrm{q} »$, lorsque le diamètre est compris entre 1.5 et $3 \mathrm{~mm}$, de type « $\mathrm{r} »$, lorsqu'elles atteignent $3-10 \mathrm{~mm}$; soit sous forme de petites opacités irrégulières, de type « $\mathrm{s}$ », lorsque leur taille est inférieure à 1,5 mm, « $\mathrm{t}$ » pour les tailles comprises entre 1.5 et $3 \mathrm{~mm}$, et « $\mathrm{u} »$ pour les tailles variant de 3 à $10 \mathrm{~mm}$. Parallèlement à la forme et à la taille, on appréciera également la densité, la profusion des images anormales, en les classant en catégories 0,1 , 2, 3, selon leur nombre croissant, en référence avec les clichés mis à la disposition des lecteurs, sachant que ces variétés de petites opacités peuvent 
coexister avec d'autres images de taille plus grande ou de conglomérats associés. Selon la forme et la taille de ces opacités plus étendues, une catégorie spécifique leur est attribuée (A, B ou C). Il peut exister, en association, des petites opacités pleurales. Quant aux autres anomalies, elles se manifestent par des coalescences, des calcifications ganglionnaires, des bulles d'emphysème ou par des anomalies au niveau du médiastin (ILO, 2011). Chez nos 2 patients, la majorité des lésions décrites ci-dessus ont été observées sauf les anomalies pleurales et les calcifications ganglionnaires (Figures 1-4). La tuberculose a été à la fois une complication et un facteur d'aggravation observé chez l'un des patients (Sujet A). La lésion radiologique n'est pas spécifique, cependant, des lésions séquellaires y sont observées (atélectasies à droite). En effet, la tuberculose est citée dans la majorité des études comme l'une des principales complications de la silicose (Ellidrissi et al., 2016 ; Elkard et al., 2016 ; Heddane et al., 2019). D'autres complications comme le pneumothorax, l'insuffisance respiratoire chronique et le cœur pulmonaire chronique sont également très fréquemment rencontrés (Heddane et al., 2019), en sachant qu'elles sont toutes observées chez nos patients.

Selon la littérature, il n'existe actuellement aucun traitement spécifique à la silicose, d'où l'importance capitale de sa prévention (Leung, Yu \& Chen, 2012 ; Centers for Disease Control and Prevention (CDCP), 2005). L'OMS recommande des mesures préventives à plusieurs niveaux : à la source, à la voie de transmission, individuelle et au niveau environnemental (Fedotov \& Eijkemans, 2007). Sharma et al. (1991) ont démontré dans une étude une amélioration significative de la fonction pulmonaire et des échanges gazeux faisant suite à une corticothérapie à type de prednisolone plus de 6 mois pour la silicose chronique. De nombreuses autres molécules, comme le citrate d'aluminium, ont été déjà proposées mais aucune d'elles n'a pu diminuer la mortalité liée à la silicose (Greenberg, Waksman \& Curtis, 2007). Ainsi, l'arrêt de l'exposition ainsi que l'identification et le traitement des comorbidités comme les infections à mycobactéries ou à d'autres germes sont d'une grande utilité (Greenberg, Waksman \& Curtis, 2007). Les individus silicotiques devraient être traités par des bronchodilatateurs et par une supplémentation en oxygène (Greenberg, Waksman \& Curtis, 2007). Pour les patients objets de cette étude dont la maladie était déjà à son stade tardif, le traitement instauré avait pour buts de soulager les symptômes. Il était à base d'oxygénothérapie, de bronchodilatateurs, de corticothérapie, de diurétique et d'anticoagulation préventive des maladies thromboemboliques, associés à une surveillance clinique régulière. Ainsi, si ces patients avaient été vus en consultation un peu plus tôt, de nombreuses complications auraient été évitées. Les particularités de ces cas sont le jeune âge des patients, les similarités de leur symptomatologie et leur lien de parenté. 
À Madagascar, des mesures pourraient être proposées pour améliorer cette situation. Le système de santé à Madagascar se doit d'élaborer une loi spécifique à propos de ces travailleurs. Il est recommandable une surveillance des conditions de travail, dans le secteur minier et de taillage de pierre et une instauration des mesures universelles de protection. Un contrôle régulier (annuel au minimum) de l'état de santé des travailleurs devrait être également systématique afin de détecter les symptômes précoces de la maladie et de prévenir les lourdes complications.

\section{Conclusion}

La silicose constitue une des plus graves affections parmi les maladies professionnelles. Pour nos deux cas, l'exposition prolongée et répétée à la silice contenue dans le quartz rose a été la source de multiples lésions, surtout, inflammatoires au niveau du parenchyme pulmonaire. Ces lésions se sont manifestées principalement par une dyspnée chronique sur le plan clinique et par des micronodules et des atélectasies sur le plan radiologique. Le traitement était essentiellement symptomatique du fait du stade avancé de la maladie. Les mesures de protection lors du travail sont d'une grande importance pour tout travailleur à risque.

Conflit d'intérêt : Les auteurs ne déclarent aucun conflit d'intérêt.

\section{References:}

1. Centers for Disease Control and Prevention (CDC) (2005). Silicosis mortality, prevention, and control--United States, 1968-2002. Morb Mortal Wkly Rep. April;54(16):401-5.

2. Elidrissi, A., Zaghba, N., Benjelloun, H., \& Yassine, N. (2016). Poumon du puisatier. Pan Afr Med J. 2016; 25: 157. DOI:10.11604/pamj.2016.25.157.10824.

3. Elkard, I., Zaghba, N., Benjelloun, H., Bakhatar, A., \& Yassine, N. (2016). La silicotuberculose. Rev Pneumol Clin. Mai;72 (3) : 179-83.

4. Fourth International Pneumoconiosis Conference (1971). Report of the Working Party on the Definition of Pneumoconiosis. Geneva, Switzerland: International Labour Organization.

5. Fedotov, A. \& Eijkemans, G. (2007). Le Programme mondial OIT/OMS pour l'élimination de la silicose (Global Programme for the Elimination of Silicosis, GPES). GOHNET (Global Occupational Health Network) Newsletter; $12: 2$.

6. Greenberg, I., Waksman, J., \& Curtis, J. (2007). Silicosis: A Review. Dis Mon. 2007 ; 53:394-416. doi:10.1016/j.disamonth.2007.09.020

7. Han, L., Yao, W., Bian, Z., Zhao, Y., Zhang, H., \& Ding, B. (2019). Characteristics and Trends of Pneumoconiosis in the Jiangsu Province, 
China, 2006-2017. Int J Environ Res Public Health. February; 16(3): 437. Doi : 10.3390/ijerph16030437.

8. Hassinet, H., Benhassine, W., Bensekhria, N., Benaicha, S., Bouhidel, W., \& Chenouf (2006). Aspects cliniques, évolutifs et radiologiques de la silicose des tailleurs de pierre. Archives Des Maladies Professionnelles et de l'Environnement. Juin 2006; 77(3) : 549. Doi:10.1016/j.admp.2016.03.450.

9. Heddane, R., Djebaili, R., Nemouchi, A., Direche, R., \& Djebbar, A. (2019). La silicose maligne chez les tailleurs de pierres. Rev Mal Respi. Janvier; 36: A131.

10. International Labour Organization (ILO) (2011). Guidelines for the use of ILO International Classification of Radiographs of Pneumoconioses. Geneva, Switzerland: ILO, 1980. Edition revised edition. Diqponible à l'URL https://www.ilo.org/wcmsp5/groups/public/---ed_protect/---protrav/--safework/documents/publication/wcms_108568.pdf

11. Leung, C., Yu, I., \& Chen, W. (2012). Silicosis. The Lancet. 2012; 379 (9830) : 2008-2018.

12. Senhaji, L., El Biaze, M., Serraj, M., Amara, M., \& Benjelloun, MC. (2015). Profil clinique, radiologique et fonctionnel des silicotiques de la mine de Jerrada. Rev Mal Respir. Janvier; 32: A153. Doi :10.1016/j.rmr.2014.10.114.

13. Sharma, Pande, J, \& Verma, K. ...(1991). Effect of Prednisolone Treatment in Chronic Silicosis. AJRCCM. Avril ;143 (4_pt_1) : 81421. Doi :10.1164/ajrccm/143.4_Pt_1.814

14. Ziskind, M., Jones, R., \& Weill, H. (1976). Silicosis1-3. American Review of Respiratory Disease. May ; 113(5) : 643-65. 